\title{
Management of Class I crowding by comprehensive edgewise orthodontic therapy
}

\author{
Mamun MSA1 BDS, Rabbani RMG ${ }^{2}$ BDS, Shupty EH ${ }^{3}$ BDS, Haq ME ${ }^{4}$ BDS, Dorth, RCS, FCPS (Hon) and \\ Hossain MZ5 BDS, PhD
}

\begin{abstract}
This case report describes the management of a 25-year-old female patient having Class I malocclusion with maxillary and mandibular crowding.The treatment include extracting the lower right central incisor and comprehensive edgewise orthodontic therapy. We decided extraction of lower right central incisor because it was broken down crown and also for shortening the treatment time and easy correction of mandibular crowding.
\end{abstract}

Keywords: crowding, mandibular incisor extraction, edgewise orthodontic therapy. (Ban J Orthod \& Dentofac Orthop, April 2011; Vol-1, No. 2, 25-28)

\section{INTRODUCTION}

Several approaches for crowded mandibular anterior teeth are currently employed: distal movement of posterior teeth, lateral movement of canines, labial movement of incisors, interproximal enamel reduction, removal of premolars, removal of one or two incisors, and various combinations of the above. Selecting the best treatment is often difficult, and all guidelines do not apply to every case. ${ }^{2}$

Treatment by extraction of one single mandibular incisor is not popular in the orthodontic profession despite the apparent advantages of the extraction in the region of crowding. 3,4 Objections to this extraction option have been based on case reports or subjective clinical opinions after observing less desirable outcomes in treated Class I and Class II malocclusions. 1,2,5,6 Unwanted sideeffects have been increases of overbite and overjet beyond acceptable limits, space reopening, partly unsatisfactory posterior occlusion, recurrence of crowding in the remaining three incisors, and unaesthetic loss of the interdental papillae in the mandibular anterior region.1,2,5-8 Kokich and Shapiro5 stated that if lower incisor extraction is performed without careful planning, the resulting occlusal discrepancy often cannot be resolved satisfactorily. They argued that with careful case selection, single incisor extraction may allow the clinicians to use simple treatment mechanics and achieve good results. A careful and realistic diagnostic setup was considered to be an important aid in determining tooth size discrepancies, and whether or not the occlusal result would be acceptable and consistent with the treatment objectives. According to Owen, ${ }^{1}$ patients who are suitable for single lower incisor extractions usually fit the following diagnostic pattern: Class I molar relationship, moderately crowded lower incisors, mild or no crowding in the upper arch, acceptable soft-tissue profile, minimal to moderate overbite and overjet, no or minimal growth potential, and missing lateral incisors or peg shaped laterals.

\section{HISTORY AND DIAGNOSIS}

A 25-year-old female came to the Department of Orthodontics and Dentofacial Orthopedics, Dhaka Dental College and Hospital, Mirpur-14, Dhaka-1206 for orthodontic treatment with the chief complaint of upper and lower crowding with lock bite on upper right and left lateral incisors and broken down crown of lower right central incisor. She was in the permanent dentition and had a symmetric face with a straight profile. She had no important dental and medical history.

Extra oral Examination showed that there was straight profile, normal vertical relation, competent lip, symmetrical face and no abnormal sign of TMJ.[fig:1]

Intraoral examination showed that there was present crowding in the upper arch and mild crowding in anterior region of the lower arch and broken down crown lower right central incisor. In occlusion, she had a $2.5 \mathrm{~mm}$ over-bite and a $1.5 \mathrm{~mm}$ over-jet and upper right and left lateral incisors were in cross-bite. There was Class I molar and canine relationships. Her maxillary midline was shifted $2 \mathrm{~mm}$ to the left. No mandibular shift was detected on closure. [fig:2]

Model analysis showed that the arch length deficiencies were (-) $6 \mathrm{~mm}$ in the maxillary arch and (-4) $\mathrm{mm}$ in the mandibular arch.

Panoramic radiographs revealed that all the permanent teeth were present except upper left wisdom and BDC lower right central incisor. [fig:3]

Cephalometric evaluation showed that she had a skeletal Class I relationship with lower lip was slightly protrusive to the "E" line. [fig: 5A]

\section{TREATMENT OBJECTIVES}

The goals of orthodontic treatment for the patient were to (1) eliminate the dental crowding in the lower and upper arch; (2) correct the cross bite; (3) correct the maxillary dental midline discrepancy; (4) maintain a Class I molar and canine relationship, (5) establish normal overjet and maintain normal overbite; (6) provide for a more regular alignment of the maxillary and mandibular teeth for aesthetics, function and structural balance.

\section{TREATMENT MECHANICS AND PROGRESSION}

Considering all aspects of the case in detail, during the treatmentplanning interview, the mandibular right central incisor and upper right first premolar were extracted, and treatment started

\footnotetext{
1,2FCPS-II Trainee, Department of Orthodontics \& Dentofacial Orthopedics, Dhaka Dental College. ${ }^{3}$ Post graduate trainee, dept. of Conservative Dentistry, Dhaka Dental College \& Hospital, ${ }^{4}$ Professor \& Head, Department of Orthodontics, City Dental College and Hospital ${ }^{5}$ Professor \& Head, Department of Orthodontics \& Dentofacial Orthopedics, Dhaka Dental College and Hospital.
} 
with a fixed appliance in the lower and upper arches (Standard edge wise $0.018 ")$. Initial levelling was accomplished with the use of 0.014" multiloop archwires over 4 months. After initial levelling, a compressed coil spring was applied to open space for upper left lateral incisor and retracted canine for upper right lateral incisor with .016-inch stainless steel archwire. The remaining right upper first premolar extraction space was closed by anchorage loss. After the opening of space, 0.014 " stainless steel archwire with 'L' loops was placed to correct the cross bite of upper lateral incisors, in that time lock was free by using posterior bite plane. In the lower arch, segments of elastomeric chain were used at the onset of treatment to close the extraction space.
Compensating bends were placed in the lower archwire to prevent excessive crown tipping at the extraction site [fig: 6]. After11 months (from the time of placing full appliances) all teeth were aligned, the extraction space was closed and midline discrepancy corrected. Then 6 months, 0.016X0.022-inch rectangular stainless steel wires were used for torque corrections, paralleling the roots, and detailing the occlusion. After satisfactory interdigitation was achieved, the fixed appliances were removed, and maxillary removable retainer and mandibular fixed lingual retainer were placed. The active orthodontic treatment time was 17 months.
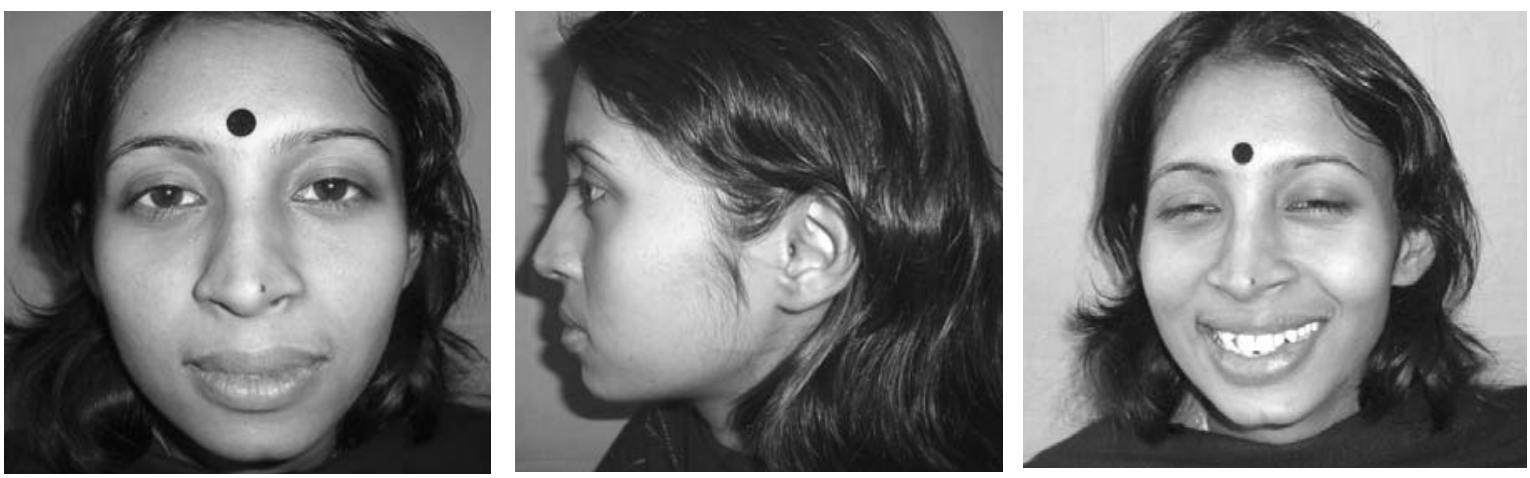

Fig: 1 Extra oral photograph (before treatment)
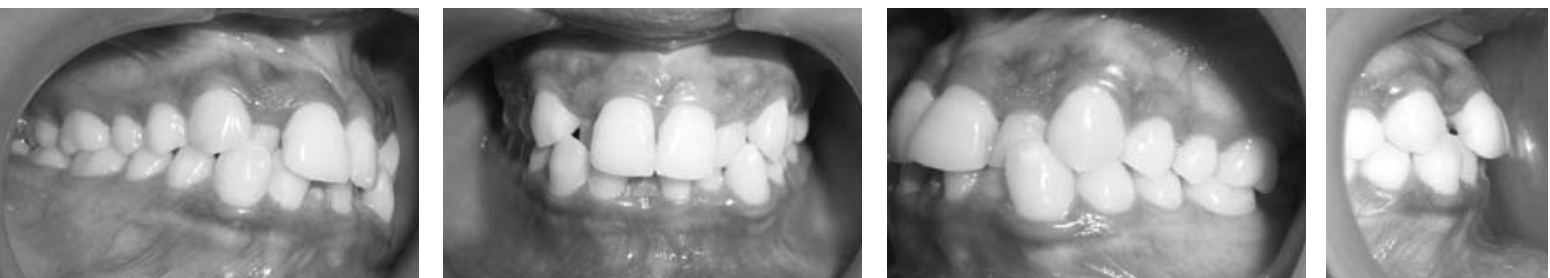

Fig: 2 Intra oral Photograph (Before Treatment)

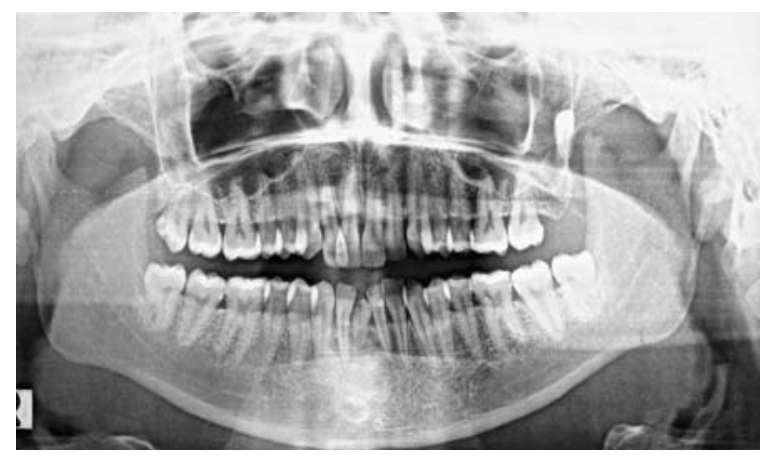

Fig: 3 Panoramic Radiograph (before treatment)

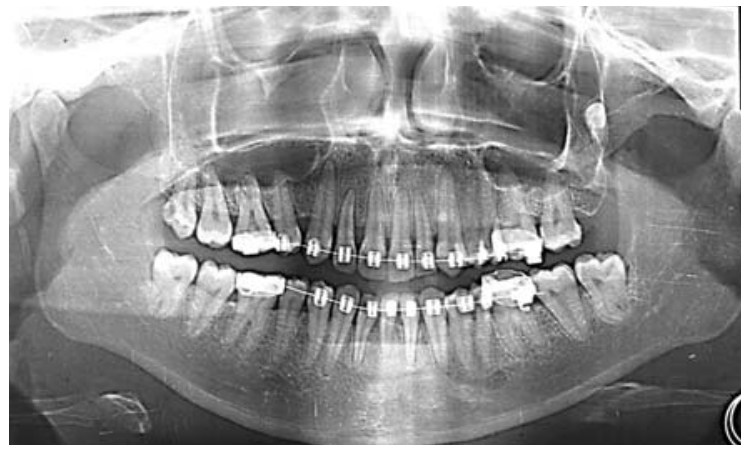

Fig: 4 Panoramic Radiograph (after treatment) 

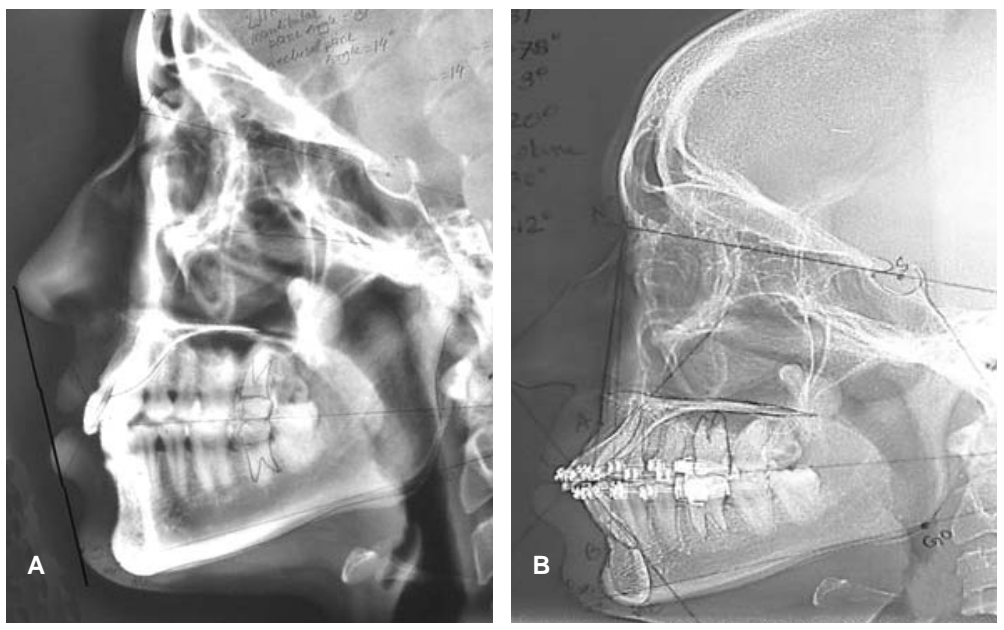

Fig: 5 Cephalogram before(A) and after treatment(B)
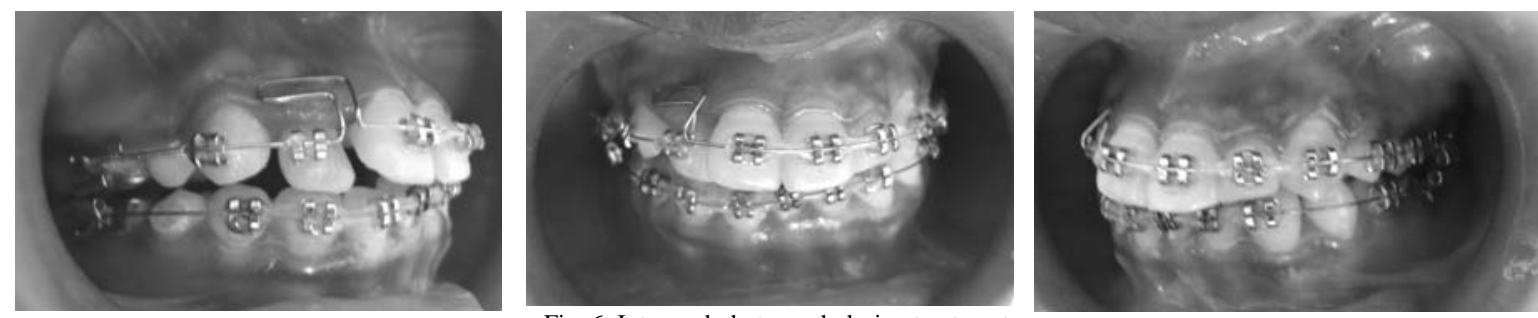

Fig. 6: Intra oral photograph during treatment
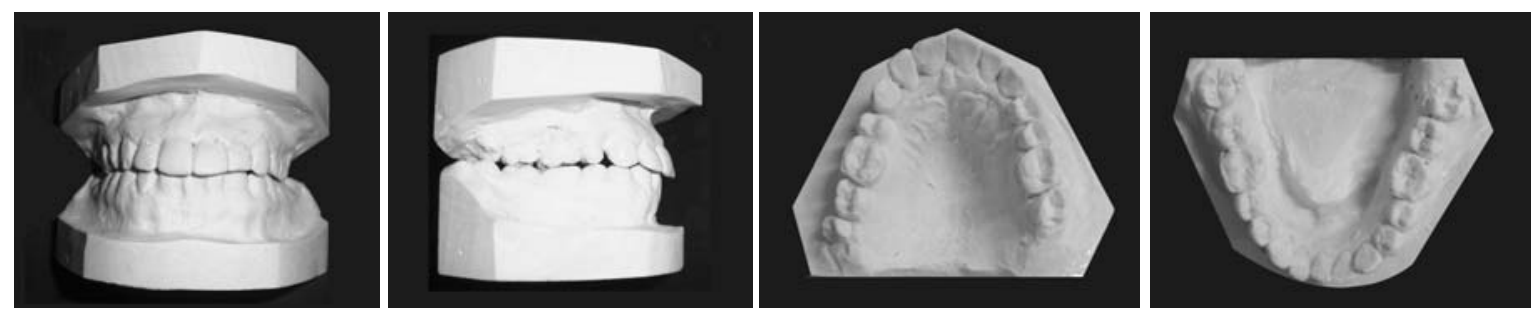

Fig. 7: Model after treatment
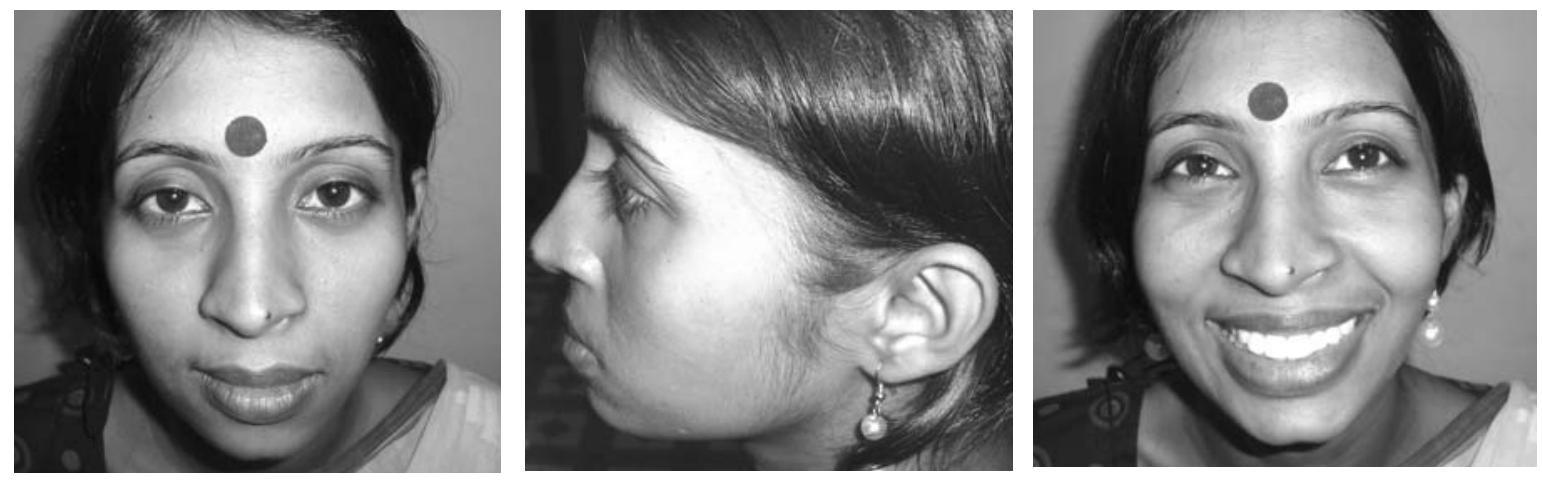

Fig. 8: Extra oral photographs after treatment

Bangladesh Journal of Orthodontics and Dentofacial Orthopedics (BJO \& DFO) 


\section{RESULTS AND DISCUSSION}

The Class I molar on left side (but right molar relationship was slight class II) and class I canine relationship were maintained with satisfactory interdigitation of posterior teeth. The overjet was established and the overbite was improved. The mandibular dental midline was become the center of the remaining lower central incisor [fig:7]. The dentition and the periodontal tissues remained healthy during treatment. Unaesthetic loss of the interdental papillae between the lower central incisors (Black triangle) was occurred as an unwanted side-effect. The smile of the patient was improved after treatment. [fig:8]

Post treatment radiographs showed that minimal root resorption had occurred during treatment and that root parallelism was satisfactory [fig:4]. Cephalometric evaluation revealed that no significant changes were occurred except the increasing of the overjet and overbite. [fig:5B].

Extraction of one mandibular incisor is generally done in patients with Bolton discrepancies greater than $2.0 \mathrm{~mm}$. The decision to extract should be supported by initial records, diagnostic wax set-up, and clinical experience. Additional information, such as Bolton analysis, shape of maxillary incisor crowns, and amount of interproximal enamel is also important. ${ }^{10}$ Reidel $^{9}$ has suggested that in patients with severely crowded mandibular arches, the removal of one or more mandibular incisor(s) is the only logical alternative which may allow for increased stability of the mandibular anterior region without continuous retention. ${ }^{11}$ In this case, we believed that treatment results would be stable because of the fact that intercanine width was decreased, and the lower incisors were not protruded.

\section{CONCLUSIONS}

One single mandibular incisor extraction is not popular in the orthodontic profession but it can be an effective treatment choice for the appropriate malocclusion with a Bolton discrepancy.
However, several factors must be considered before making the final treatment decision. In addition, evaluation of a diagnostic wax set-up will allow the orthodontist to predict the success of the proposed treatment plan.

\section{REFERENCES}

1. Owen AH. Single lower incisor extractions. J Clin Orthod. 1993;27:153-160.

2. Valinoti JR. Mandibular incisor extraction therapy. Am J Orthod Dentofac Orthop. 1994;105:107-116.

3. Gottlieb E, Nelson AH, Vogels DS. JCO study of orthodontic diagnosis and treatment procedures. Part I: Results and trends. J Clin Orthod. 1996;30:615-629

4. Travess H, Harry DR, Sandy J. Orthodontics. Part 8: Extraction in orthodontics. Br Dent J. 2004;196:195-203

5. Kokich VG, Shapiro PA. Lower incisor extraction in orthodontic treatment: four clinical reports. Angle Orthod. 1984;54:139-153.

6. Klein DJ. The mandibular central incisor, an extraction option. Am J Orthod Dentofac Orthop. 1997;111:253-259.

7. Dacre JT. The long term effects of one lower incisor extraction. Eur J Orthod. 1985;53:706-713.

8. Canut JA. Mandibular incisor extraction: indications and long-term evaluation. Eur J Orthod. 1996;18:485-489.

9. Riedel RA, Little RM, Bui TD. Mandibular incisor extraction: postretention evaluation of stability and relapse. Angle Orthod. 1992;62:103-116

10. Kokich VO. Treatment of a Class I malocclusion with a carious mandibular incisor and no Bolton discrepancy. Am J Orthod Dentofac Orthop. 2000;118:107-113

11. Blake M, Bibby K. Retention and stability: A review of the literature. Am J Orthod Dentofac Orthop. 1998;114:299-306.

\section{Correspondence}

Dr. Mohammad Shamim Al Mamun BDS FCPS-II Trainee,

Dept. of Orthodontics and Dentofacial Orthopedics

Dhaka Dental College and Hospital, Mirpur-14, Dhaka-1206

Email: mamun_d36@yahoo.com , Cell: +8801712718527 